\title{
Determinación del intervalo postmortem mediante el estudio de la sucesión de insectos en dos cadáveres hallados en el interior de una finca rústica en Madrid.
}

Assessment of post-mortem interval through the study of
the insect succession in two corpses found indoors in a
country house in Madrid.

A.M. García-Rojo', L. Honorato', M. González y A. Téllez

RESUMEN

En este artículo se presenta un caso estudiado en el Laboratorio de Entomología Forense de la Sección de Antropología de la Comisaría General de Policía Científica (Cuerpo Nacional de Policía). En él, se analizaron muestras entomológicas recogidas en dos cadáveres durante la práctica de la Inspección Ocular Técnico Policial, llevada a cabo en la localidad de Fuenlabrada, sita en la zona Sur de Madrid (España, Región Paleártica). Los dos cadáveres, en avanzado estado de descomposición, fueron encontrados en el interior de una finca rústica en invierno del año 200I. Las evidencias de interés forense recogidas consistieron en dípteros de las Familias Calliphoridae, Muscidae y Phoridae y Coleoptera de las Familias Cleridae, Dermestidae e Histeridae. Además, arácnidos que permitieron afirmar la compatibilidad de los especímenes con el entorno. La sucesión de los insectos recogidos permitió estimar el intervalo post-mortem en un año.

Palabras clave: Entomología Forense, sucesión de insectos, intervalo post-mortem, Madrid, España.

Cuad Med Forense 2009; 15(56):137-145

\section{ABSTRACT}

In this paper, it is reported a case which was studied in the Forensic Entomology Laboratory belonging to the Anthropology Section from the General Department of the Scientific Police (Spanish National Police). We analysed entomological evidences collected during death scene investigation performed in Fuenlabrada, south of Madrid administrative region (Spain, Paleartic Region). Both corpses, in advanced decomposition stage, were found indoors in a country house in winter 200I. Samples collected consisted of Diptera identified as Calliphoridae, Muscidae and Phoridae Families; Coleoptera corresponding to Cleridae, Dermestidae and Histeridae ones. Furthermore, spiders which allowed us to confirm the compatibility with the place where carcasses were found. The study of the insect succession allowed the estimation of post-mortem interval in one year.

Key words: Forensic Entomology, insect succession, Postmortem interval, Madrid, Spain.

Fecha de recepción: 4.FEB.09

Fecha de aceptación: 28.MAY.09

Correspondencia: A. M. García-Rojo. E-mail: ana.garcia@dgp.mir.es. Teléfono: 9158283 66; Fax: 913003521

1 Sección de Antropología de la Comisaría General de Policía Científica (Cuerpo Nacional de Policía). Dirección General de la Policía y de la Guardia Civil. Ministerio del Interior. Madrid. España. 


\section{INTRODUCCIÓN:}

La "Entomología Médico Criminal" o Entomología Forense es una disciplina incluida en las Ciencias Forenses que utiliza los datos que los artrópodos pueden aportar en la investigación médicolegal de la muerte, especialmente en aquellos casos de muerte violenta [I].

La entomología forense, por otro lado, proporciona una fuerte evidencia científica que puede ser defendida con éxito. Tiene diversas aplicaciones de muchísimo interés. La entomotoxicología, mediante la que se pude determinar la presencia de sustancias tóxicas, venenos, estupefacientes en estadíos pre-imaginales y adultos de insectos de interés forense [2]. Asimismo, se encarga del estudio de los efectos causados por estas sustancias en el desarrollo de los artrópodos [3] y de la miasis o infestación de vertebrados vivos por larvas de dípteros que, durante al menos un periodo de tiempo, se alimentan de tejidos muertos o vivos, fluidos corporales o el alimento ingerido por el huésped. En casos de negligencia o maltrato deliberado, el análisis entomológico puede llegar a ser una poderosa evidencia para datar el periodo de tiempo de abuso o negligencia [4]. Otra de las posibles aplicaciones de la entomología forense al ámbito policial se observa en la detección y determinación de residuos de disparo en estadíos pre-imaginales de diferentes especies de dípteros, aunque se ha avanzado poco en este sentido. Además la entomología molecular forense se encarga del análisis de estadíos pre-imaginales y adultos de hexápodos de interés forense, mediante técnicas de ADN nuclear y ADN mitocondrial. El valor de los estudios moleculares reside en utilizar la citada tecnología ADN, para identificar poblaciones geográficas de dípteros, permitiendo de esta manera al taxónomo comentar los posibles movimientos de los que pudiera haber sido objeto el cadáver; además de la correcta y rápida identificación de los artrópodos. [5,6,7]. Cuando no se dispone de los restos cadavéricos para la extracción de ADN, esta extracción puede realizarse del contenido intestinal de los estadíos larvarios de los insectos que se alimentan del cadáver [8].

Una de las aplicaciones más habituales de la Entomología Forense es la estimación del intervalo post-mortem (IPM).

Siguiendo la nomenclatura recomendada por los entomólogos forenses internacionales, el proceso de putrefacción cadavérica se divide en cinco fases denominadas: Cadáver fresco, hinchado, descomposición activa, descomposición avanzada y esqueletización.

Cuando el cadáver está en avanzado estado de putrefacción, la estimación de la data de la muerte se basa en el estudio de la sucesión de insectos que acuden al cuerpo muerto, atraídos por la materia orgánica en descomposición o por diversos productos derivados de la putrefacción. En este artículo se presenta el estudio de la sucesión de insectos en dos cadáveres en avanzado de putrefacción encontrados en el interior de una finca rústica en la localidad de Fuenlabrada (Madrid). Los datos obtenidos permitieron estimar un IPM de un año.

Para que los resultados obtenidos puedan ser aceptados plenamente en los Tribunales de Justicia es necesario impulsar el desarrollo de un sistema de calidad. En la actualidad es la Asociación Europea de Entomología Forense, (EAFE), creada en el año 2002, la que dicta estos criterios, con la publicación de una serie de recomendaciones y estándares básicos en el año 2007 [9], sobre el trabajo de campo, la prevención de la contaminación o destrucción de evidencias y garantizar la cadena de custodia, además del estudio y tratamiento de muestras en el laboratorio. Hasta la implantación de los criterios de calidad, anteriormente citados, se trabajaba de acuerdo a los protocolos de Lord \& Burger (1983) [10], aplicados en este estudio, que data del año 2001 . 


\section{EXPOSICIÓN DEL CASO:}

En Noviembre de 200 I se hallaron los cadáveres de un hombre y una mujer, en avanzado estado de descomposición, situados en el interior de una finca rústica ubicada en un descampado al este de la localidad de Fuenlabrada, coordenadas UTM X: 435399.67, Y:4459644.78, Huso 30, en la zona sur de la Comunidad Autónoma de Madrid (Fig. I y 2).

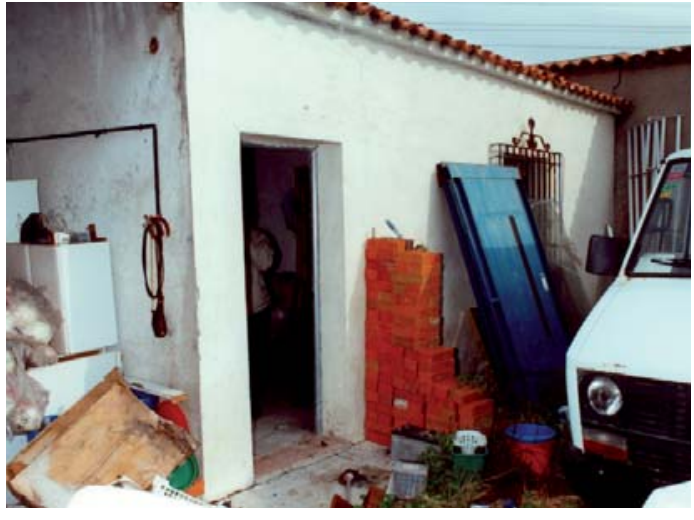

Fig. 1.- Finca rústica donde se encontraron los cadáveres.

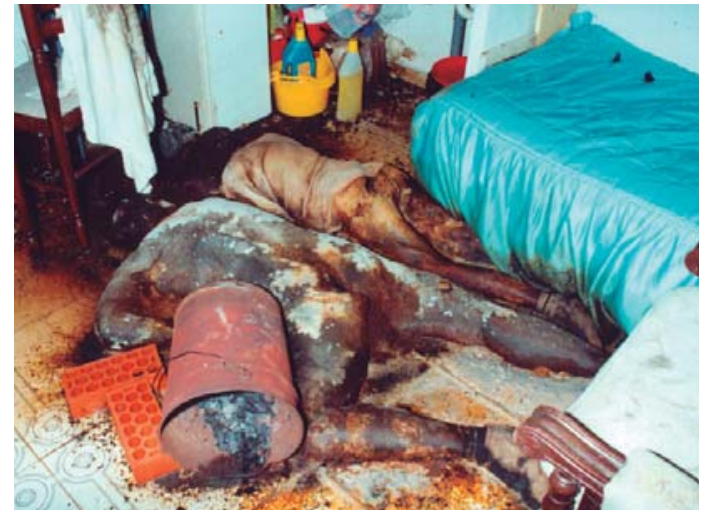

Fig. 2.- Localización de los cadáveres en el interior de la vivienda.

En el interior de la finca estaba la parte construida para vivienda y su prolongación habilitada con tejado para garaje. La zona destinada a vivienda era de unos seis metros de largo por tres de ancho y disponía de una puerta metálica. Franqueada la puerta había una habitación que no tenía ventana y un tubo dispuesto en la salida de gases de calderas que se encontraba obturado intencionadamente. A la derecha de esta habitación había otra dependencia de dimensiones similares con una ventana con un pequeño cristal fracturado.

Al abrir la puerta metálica de acceso a la vivienda, en la habitación principal, se encontraron en el suelo dos cadáveres tendidos decúbito prono en fase de descomposición activa. Uno de los cadáveres correspondía a un varón que presentaba la parte expuesta al aire, es decir, la espalda, momificada. En la mano izquierda faltaba todo el tejido conjuntivo y epidermis de las falanges distales, a excepción del dedo pulgar. El otro cadáver era el de una mujer a la que faltaban partes musculares. Se observó una gran cantidad de insectos en distintos estadíos de desarrollo, tanto en los cadáveres como a su alrededor (Fig. 3) lo que, probablemente, contribuyó a la destrucción de masa muscular y partes blandas de los mismos (Fig. 4).

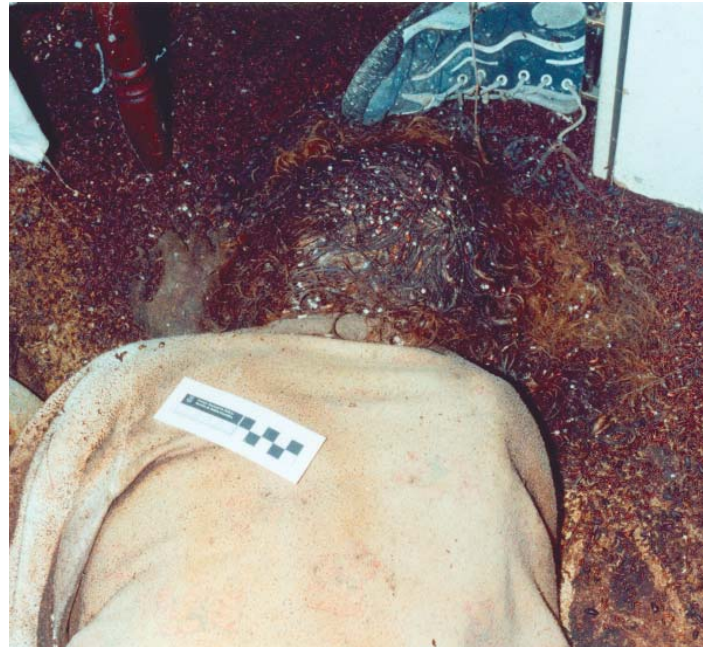

Fig. 3.- Cadáver en el que se observan gran cantidad de insectos.

Aunque el lugar estaba cerrado, impidiendo la colonización de los artrópodos de interés forense, podrían haber accedido al recurso trófico a través de cualquier pequeño orificio abierto, como el cristal fracturado de la ventana de una de las habitaciones de la citada vivienda. 


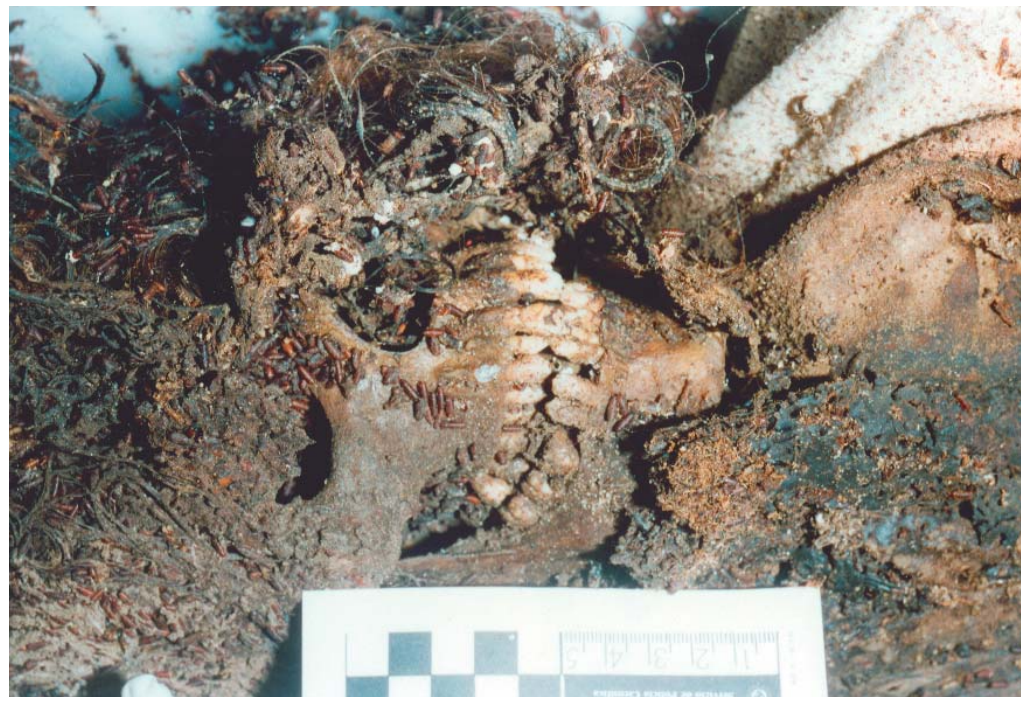

Fig. 4.- Ausencia de partes blandas por la acción de los insectos.

Hay que destacar que uno de los cadáveres, el más cercano a la puerta, tenía junto a su brazo izquierdo un bidón metálico de unos 25 litros, caído, en cuyo interior se encontró carbón vegetal procedente de fuego o brasas de madera. En este sentido, unos conocidos de los fallecidos, que habían estado conviviendo con ellos durante un tiempo, declararon que cuando hacían fuego metían las ascuas en una lata para calentarse y no abrían para ventilar, impidiendo de esa manera el acceso a los roedores. En una ocasión, la pareja salió mareada a causa de la inhalación de monóxido de carbono.

\section{MATERIAL Y MÉTODO:}

\section{RECOGIDA DE EVIDENCIAS}

Durante la práctica de la Inspección Ocular Técnico Policial, realizada por expertos del Grupo de Policía Científica de esa localidad, se recogieron las muestras de insectos que se detallan a continuación, algunos vivos, que fueron fotografiados, etiquetados y conservados "in situ" conforme al protocolo adoptado por la Comisaría General de Policía Científica siguiendo el criterio de Lord \& Burger (1983) [10]. Se tomaron de la zona donde se encontraba la cabeza del varón y de la mujer respectivamente, y de entre las cabezas de los dos cadáveres, puparios de diptero, de distinto tamaño y morfología, algunos de ellos abiertos. De entre los restos viscosos situados debajo y alrededor de los fallecidos, se recogieron larvas, puparios y adultos muy fragmentados de díptero, larvas, élitros, exuvias y adultos de coleóptero.

Del techo, cerca de la arista formada por las paredes de la vivienda, se tomaron artrópodos correspondientes a arácnidos.

Los imagos muertos y puparios abiertos se metieron inmediatamente en frascos de vidrio con alcohol rebajado al 70\%. Se tomaron muestras representativas de estadíos inmaduros correspondientes a larvas, mediante el empleo de pinzas y pinceles entomológicos. Al disponer de gran cantidad de muestra, una parte de las larvas se mantuvo viva. Los ejemplares se introdujeron en cajas de plástico con un sustrato de papel celulosa para poder ser criados en el laboratorio hasta la emergencia del adulto. Esta misma operación se realizó con los puparios cerrados. 
La otra parte de muestra correspondiente a larvas se escaldó durante 2 minutos y se pasó inmediatamente a frasco con alcohol rebajado al 70\%. Esta operación resulta ser muy importante para evitar la retracción del tejido larvario y el oscurecimiento de los ejemplares.

Seguidamente, fueron precintados y remitidos el mismo día, junto con el "Acta de Recogida de Muestras", copia del "Acta de Inspección Ocular Técnico Policial" y reportaje fotográfico del lugar de los hechos, al Laboratorio de Entomología Forense de la Sección de Antropología para su estudio y estimación del IPM. Todos estos requisitos son de obligado cumplimiento para asegurar la cadena de custodia de las muestras.

Es preciso destacar que la identidad de los fallecidos se realizó mediante regeneración del tejido dactilar de los pulpejos de los dedos extraídos en la autopsia, por lo tanto, no fue necesario realizar ningún estudio antropológico o de ADN.

\section{TRATAMIENTO DE MUESTRAS EN EL LABORATORIO}

En el laboratorio, las muestras correspondientes a puparios y larvas de dípteros que estaban vivas fueron criadas en condiciones estandarizadas, en cámara de cultivo, marca Heraeus, modelo B-12, hasta obtener el adulto.

Además, las larvas de dípteros que se recibieron muertas fueron montadas en preparaciones microscópicas y se realizó el estudio morfológico de las estructuras presentes en el segmento anal y parte anterior de las mismas.

A posteriori, se procedió a la identificación de los insectos, etiquetado y almacenaje. Todos ellos se conservaron adecuadamente conforme a protocolo estandarizado [I l]. Es preciso señalar que la identificación específica de los imagos (Diptera: Muscidae) fue realizada por el Dr. Adrian Pont( ${ }^{(1)}$, y la de los arácnidos por el Dr. Antonio Melic(2).

\section{RESULTADOS:}

El estudio realizado en el Laboratorio de Entomología Forense permitió obtener los siguientes datos acerca de la sucesión de insectos:

- Larvas (Fig. 5), puparios abiertos, vacíos de contenido y algunos cerrados (Diptera: Muscidae) (Fig. 6). Es preciso señalar, que esta muestra es la que contenía el número más elevado de ejemplares.

- Adultos muy fragmentados y deteriorados (6 machos y 2 hembras) de la especie Hydrotaea capensis (Wiedemann, 1818) (Diptera: Muscidae), antes denominada Ophyra capensis (Fig. 7).

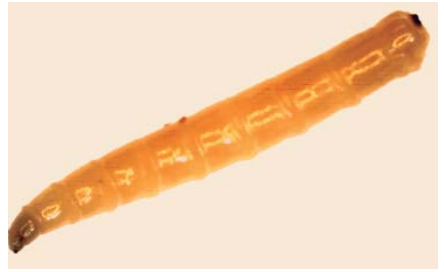

Fig. 5.- Larvas (Diptera: Muscidae)

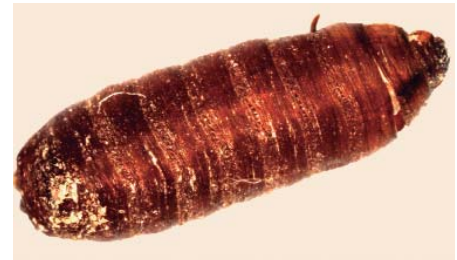

Fig. 6.- Puparium (Diptera:Muscidae).

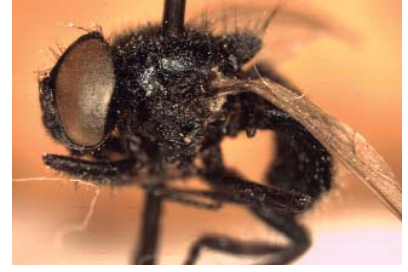

Fig. 7.- Hydrotaea capensis (Wiedemann, 1818) (Diptera: Muscidae)

(1) Informe del Dr. Adrian Pont, experto en múscidos, de fecha 27 de febrero de 2002.

(2) Informe del Dr. Antonio Melic, experto en arácnidos, de fecha 10 de diciembre de 2001. 
- Puparios, abiertos y vacíos de contenido (Diptera: Fanniidae).

- Dos puparios cerrados de Diptera de la familia Calliphoridae.

- Puparios abiertos y vacíos de contenido (Diptera: Phoridae).

- Dos arañas (hembras) de la especie Steatoda triangulosa (Walckenaer, 1802).

- Larvas oligópodas de Coleoptera, Familia Cleridae.

- Élitros e imagos completos de la especie Necrobia rufipes (De Geer, 1775) (Coleoptera: Cleridae).

- Exuvias correspondientes a larvas de Derméstidos, además de un imago de la especie Dermestes frischi (Dermestinus) Kugelann 1792 (Coleoptera: Dermestidae).

- Un imago de la especie Carcinops (Carcinops) pumilio Erichson, 1834 (Coleoptera: Histeridae).

\section{DISCUSIÓN:}

Los distintos estadíos de desarrollo que conforman el ciclo biológico de la especie Hydrotaea capensis suelen aparecer de junio a octubre, siendo más abundantes en agosto [I 2]. Las larvas se hacen depredadoras en el estadío de desarrollo III y atacan a otras larvas que viven en el mismo medio $[13,14,15,16]$. Esta especie, junto con $H$. ignava, coloniza el cadáver después de Calliphora y Muscina pero antes que los fóridos [17].

La especie Hydrotaea capensis se siente fuertemente atraída por el olor a amoniaco desprendido en la fase de fermentación amoniacal. La presencia de estos dípteros nos indicaría que este lugar pudiera ser seco y cálido. Según Smith [15], esta especie prefiere un ambiente más cálido que Ophyra leucostoma. Este autor observó la presencia de Hydrotaea capensis asociada a cadáveres que se habían mantenido en interior durante meses, donde los califóridos de primeras generaciones no suelen tener acceso. Suele ser común en zonas rurales donde se alimenta junto a otros múscidos, entre los que se encontrarían aquellos de la Familia Fanniidae. La presencia de especímenes en distintos estadíos de desarrollo de fánidos no tiene valor diagnóstico [18] para la determinación del IPM pero, en el caso que nos ocupa, su presencia es compatible con el entorno en que fueron hallados los cadáveres.

Es preciso señalar que la especie Hydrotaea capensis fue detectada en dos casos analizados en el Laboratorio de Entomología Forense de la Sección de Antropología de la Comisaría General de Policía Científica. El primero de ellos, en relación con el hallazgo de dos cadáveres en interior de vivienda en la localidad de Alicante. En el segundo, se recibió con la entomofauna recogida en un cadáver encontrado en el interior de una bodega en Málaga. En ambos casos se estimó un IPM de un año.

Por otra parte, los puparios abiertos y vacíos de contenido (Diptera: Muscidae) indicarían que ha habido numerosas generaciones de moscas que podrían haber penetrado desde el exterior, cuando las condiciones ambientales fueran las adecuadas (primavera-verano, especialmente en agosto).

Los puparios cerrados (Diptera: Calliphoridae) (Fig. 8) fueron los especímenes recogidos en menor número. Estos dípteros suelen acceder al cadáver en la fase inicial de descomposición, devorando tejido blando y húmedo. La escasez de estos elementos indicaría que el cuerpo no ha estado accesible a estos dípteros en las fases iniciales. Esto podría ser debido al difícil acceso de los individuos adultos desde el exterior y por lo tanto indicaría que los cadáveres podrían haber permanecido, desde la muerte hasta el hallazgo, en el interior de la vivienda. 
En la mayor parte de los casos en los que se estima el IPM mediante el estudio entomológico forense, intervienen Califóridos y otros Calyptrata [19]. Cuando éstos hexápodos no pueden colonizar un cadáver, son los Fóridos, entre otros, los que acceden al recurso trófico, porque pueden atravesar pequeños orificios e incluso penetrar varios metros de profundidad en el suelo [20]. Se desarrollan en un amplio espectro de materia orgánica en descomposición. Pueden acudir al cuerpo muerto en cualquier momento, encontrándose normalmente entre las primeras oleadas de insectos que se encuentran en el cadáver [2I]. Campobasso et al. [22] exponen que los dípteros de la Familia Phoridae pueden aparecer en estadíos iniciales de putrefacción, asociados a otras especies de moscas, como ocurre en el caso que nos ocupa.

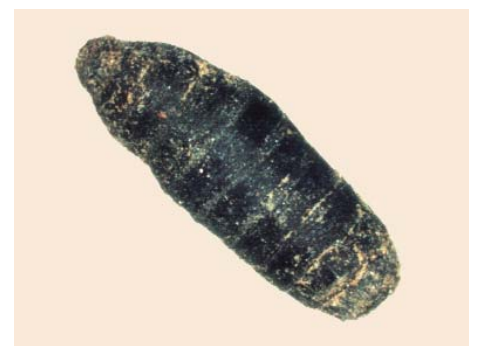

Fig. 8.- Puparium cerrado (Diptera: Calliphoridae)

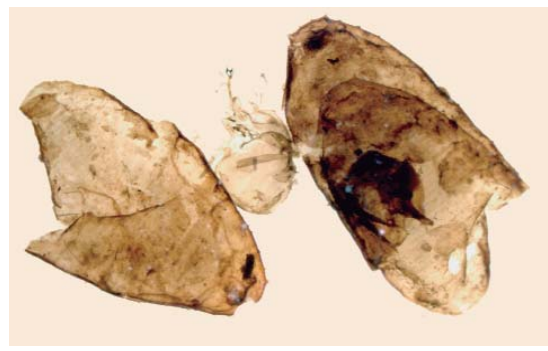

Fig. 9.- Puparium (Diptera: Phoridae)

La presencia de los cléridos de la especie Necrobia rufipes y derméstidos de la especie Dermestes frischi son compatibles con el estadío de putrefacción avanzado de los cadáveres. La presencia de larvas, exuvias y adultos de estos coleópteros, indicaría que, al menos, ha habido una generación sobre los cadáveres. Esto representaría una infestación del recurso, que podría ser útil para la estimación del IPM [23].

La especie Necrobia rufipes suele encontrarse en cadáveres prácticamente esqueletizados, alimentándose de otros insectos [23]. Aunque los adultos son depredadores de huevos y larvas, a veces, se alimentan del cadáver [24]. Payne y King encontraron ejemplares de la especie citada alimentándose del cuerpo muerto en trampas que contenían huesos secos de cerdo [15]. Por otro lado, la presencia de la especie Dermestes frischi, cosmopolita (3), está asociada a carne seca de vertebrados ya que, en general, no se alimentan de carne fresca $[15,25]$.

La presencia de histéridos supone un control biológico importante sobre la densidad de población de dípteros, por su comportamiento depredador de huevos y larvas de moscas [26]. En el caso que nos ocupa se detectó la presencia de un imago de la especie Carcinops pumilio, cosmopolita, que vive en la Península Ibérica e Islas Baleares y abunda en cadáveres secos [27].

La presencia de arañas (dos hembras) de la especie Steatoda triangulosa (Walckenaer, I802) es bastante común y se encuentra repartida en Centroeuropa y también en la Península Ibérica. Es una especie antrópica que suele aparecer en ámbitos domiciliarios y en el interior de construcciones de todo tipo.

Analizados los datos de temperatura, que fueron tomados durante cinco días en el interior de la vivienda por funcionarios adscritos al Grupo Local de Policía Científica, se consideró que la temperatura en la habitación donde se encontraron los cadáveres era constante y que oscilaba alrededor de unos $15^{\circ} \mathrm{C}$.

(3) Informe del Dr. Michael Geisthardt, especialista en derméstidos, de fecha 3 de junio de 2002. 


\begin{tabular}{|c|c|c|c|}
\hline MUESTRAS & \begin{tabular}{|l|l|}
\multicolumn{2}{|c|}{ DESC } \\
INICIAL \\
F. \\
\end{tabular} & \begin{tabular}{|l|l|l|} 
OMPOSICIÓN \\
\multicolumn{2}{|c|}{ AVANZADA } \\
D.AC. D.AV & ESQ \\
\end{tabular} & ENTORNO \\
\hline Hydrotaea capensis Wiedemann, 1818 (Diptera: Muscidae) & & & c \\
\hline (Diptera:Calliphoridae) $)^{(1) *(*)}$ & & & c \\
\hline$(\text { Diptera:Fanniidae })^{(*)}$ & & & c \\
\hline 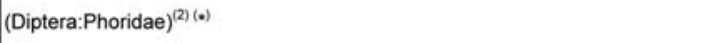 & & & c \\
\hline Necrobia rufipes De Geer, 1775 (Coleoptera: Cleridae) & & & c \\
\hline Dermestes frischi (Dermestinus) Kugelann, 1792 (Coleoptera: Dermestidae) & & & c \\
\hline Carcinops (Carcinops) pumilio Erichson 1834 (Coleoptera: Histeridae). & & & c \\
\hline Steatoda triangulosa Walckenaer 1802 & & & c \\
\hline
\end{tabular}

\begin{tabular}{|l|l|l|}
\hline $\mathrm{F} .=$ FRESCO & & DETERMINANTE PARA EL IPM \\
\hline H. $=$ HINCHADO & & POCO DETERMINANTE PARA EL IPM \\
\hline D. AC $=$ DESCOMPOSICIÓN ACTIVA & & SIN INTERÉS PARA EL IPM \\
\hline D. AV $=$ DESCOMPOSICIÓN AVANZADA & C & COMPATIBLE CON EL ENTORNO \\
\hline ESQ $=$ ESQUELETIZADO & & \\
\hline
\end{tabular}

Tabla 1: Especimenes de interés forense y cronología de aparición.

(1) Escaso número de ejemplares debido al difícil acceso al recurso.

(2) Aunque pueden acudir al cadáver en cualquier momento, prefieren los estadios iniciales.

$\left.{ }^{*}\right)$ Especimenes de interés forense de las familias citadas.

\section{CONCLUSIÓN:}

Los hallazgos entomológicos parecen sugerir que los cuerpos muertos podrían haber permanecido de forma prolongada en el mismo lugar de los hechos, no habiendo datos entomológicos que orienten en sentido contrario. Teniendo en cuenta el lugar donde fueron hallados los cadáveres (interior de vivienda), la temperatura descrita y los datos obtenidos del estudio de la sucesión de insectos, se estimó que la muerte pudo haber ocurrido a finales de verano o en otoño del año anterior, 2000. Se concluye, pues, que el intervalo de tiempo comprendido entre el momento en que se produjo la muerte de los dos individuos y su hallazgo posterior es de aproximadamente un año. Este extremo coincide también con las observaciones realizadas por Mégnin [28], que encontró Ophyra en un elevado número de cadáveres de un año. Ophyra capensis no es un díptero de fenología temprana [17] y tiene una gran importancia forense por su relación estrecha a hábitats urbanos [24]. Comúnmente denominada "mosca de los cadáveres", tal y como Adrian Pont, experto en múscidos, hace constar en el informe elaborado con motivo de este trabajo.

Posteriormente, y en el transcurso de las investigaciones, se tuvo conocimiento a través de declaraciones testificales de familiares y conocidos que la última vez que habían tenido conocimiento de ellos en vida había sido en el mes de Noviembre del año anterior. Lo que coincide con los resultados obtenidos en este trabajo en cuanto a la estimación de la data de la muerte.

\section{AGRADECIMIENTOS:}

Al Dr. Adrian Pont, especialista en múscidos, por su inestimable ayuda en la identificación de los especimenes recibidos. Al Dr. Antonio Melic, experto en arácnidos, por su asesoramiento e identificación de los dos ejemplares recogidos en la Inspección Ocular Técnico Policial.

Al Dr. Michael Geisthardt, especialista en derméstidos, por sus aportaciones en cuanto a la ecología de estos especimenes. 


\section{BIBLIOGRAFÍA:}

I.-Hall RD. Medico criminal entomology. In Entomology and Death: A Procedural Guide, Catts, E.P. and N.H. Haskell, Eds., Joyce's Print Shop, Clemson, SC, 1990.

2.-Nuorteva P.; Nuorteva SL. The fate of mercury in sarco-saprophagous flies and in insects eating them. Ambio 1982; II:34-7

3.-Sohal RS, Lamb RE. Storage excretion of metallic cations in the adult housefly, Musca domestica. J Insect Physiol 1979; 25:I19-24 4.-Benecke M, Josephi E, Zweihoff R. Neglect of the elderly: forensic entomology cases and considerations. Forensic Sci. Int. 2004; 146 Suppl: S195-9.

5.- Malgorn Y. \& Coquoz, M. (1999). "DNA typing for identification of some species

of Calliphoridae. An interest in forensic Entomology". Forensic Science International 102:III-II9.

6.- Benecke, M. "Random amplified polymorphic DNA (RAPD's) typing on necrophageous insecta (Diptera, Coleoptera) in criminal forensic studies: validation and use in practice." Forensic Science International (1998) 98:157-168.

7.- Wells, J.D. and Sperling, F.A.H. (200I): "DNA-based identification of forensically important Chrysomyinae (Diptera: Calliphoridae)." Forensic Science International I20: II0-II5.

8.-Lord W.D., Dizinno J.A., Wilson M.R., Budowle B., Taplin D. and Meinking T.I., "Isolation, amplification and sequencing of human mitochondrial DNA obtained from human crab louse, Pthirus Pubis (L.), blood meals”. J.Forensic Sci. 43 (1998) I097-II00.

9.- Jens Amendt, CarloP.Campobasso, Emmanuel Gaudry, Christian Reiter, Helene N.LeBlanc, MartinJ.R.Hall. Best practice in forensic entomology:standards and guidelines. Int.J.Legal Medicine 121:90-104. 10.-Lord WD, Burger JF. Collection and preservation of forensically important entomological materials. J Forensic Sci 1983;28(4):936944.

II.-Haskell NH, Hall R, Cervenka V], Clark MA. On the body: insect's life stage presence and the post-mortem artefacts. In WD Haglund and MA Sorg (eds), Forensic Taphonomy: the post-mortem fate of human remains, CRC Press, Boca Raton, Fl 1997; pp. 415-418.

12.-Graham-Smith GS. "Observations on the habits and parasites of common flies”. Parasitology (Cambridge) 1916;8: 440-544.

13.-Henning W. Muscidae. In Lindner ME. (ed): Die Fliegen der Palaearktischen Region, 196I - 1962, 63b. 7:461 - 620.
14.-Skidmore P. The biology of the Muscidae of the World. Dr. W. Junk Publishers. Dordrecht/ Boston/ Lancaster. xiv , 1985.

I5.-Smith KGV. A manual of Forensic Entomology. London. The Trustees of the British Museum (Natural History). 1986.

16.-Fuller ME. The insects inhabitants of carrion: A study in animal Ecology. Council for Scientific and Industrial Research 1934;, Bulletin 82:I-63.

17.-Easton AM, Smith KG. The entomology of the cadaver. Medicine, Science and Law 1970. 208-215.

18.-Benecke M. Six forensic entomology cases: description and commentary. J Forensic Sci 1988;43:797-805.

19.-Greenberg B, Kunich JC. Entomology and the law. Cambridge University Press, Cambridge, 2002.

20.-Disney RHL. Scuttle flies: the Phoridae. Chapman \& Hall, London, 1994.

21.-Oliva A. Insectos de interés forense en Buenos Aires (Argentina). Primera lista ilustrada y datos bionómicos. Revista del Museo Argentino de Ciencias Naturales "Bernardino Rivadavia" e Instituto Nacional de Investigación de las Ciencias Naturales. Entomología 1997; 7.

22.-Campobasso (P, Disney RH, Introna F. A case of Megaselia Scalaris (Loew) (Diptera:Phoridae) breeding in a human corpse. Aggrawal's Internet Journal of Forensic Medicine and Toxicology 2004:5(I): 3-5.

23.-Kulshrestha P, Satpathy DK. Use of beetles in forensic entomology. Forensic Sci Int 2001;120:15-17.

24.-Byrd JH, Castner JL. Forensic Entomology: the utility of arthropods in legal investigations. CRC Press LLC, Boca Raton, Fl $200 \mathrm{I}$. 25.-Schroeder H, Klotzbach H, Oestelhelweg L, Püschel K. Larder beetles (Coleoptera, Dermestidae) as an accelerating factor for decomposition of a human corpse. Forensic Sci Int 2002;|27:231-236. 26.-Nourteva P. Histerid beetles as predators of blowflies (Diptera: Calliphoridae) in Finland. Ann Zool Fennici 1970;7:195-198.

27.- Yelamos T. Coleoptera, Histeridae. En: Fauna Ibérica, vol.I7. Museo Nacional de Ciencias Naturales. CSIC. Madrid, 2002.

28.- Mégnin JP. La fauna des cadarres; application de I'Entomologie a la Medicine Legale; Enciclopedie Scientifique des Aides-Memoire; Masson et Gauthiers-Villars. Paris. France, 1894. 